\title{
Pericyte degeneration and acellular capillaries are increased in the feet of human diabetic patients
}

\author{
R. G. Tilton ${ }^{1}$, A. M. Faller ${ }^{1}$, J. K. Burkhardt ${ }^{1}$, P. L. Hoffmann' ${ }^{1}$, C. Kilo ${ }^{2}$ and J. R. Williamson ${ }^{1}$ \\ Departments of ${ }^{1}$ Pathology and Internal Medicine ${ }^{2}$, Washington University School of Medicine, St. Louis, Missouri, USA
}

\begin{abstract}
Summary. Ultrastructural morphometry was used to quantify capillary basement membrane width, pericyte coverage of capillaries, pericyte degeneration, and the extent of acellular capillaries in skeletal muscle obtained at autopsy from neck, thigh, calf and foot of five male and four female diabetic subjects and an equal number of sex- and age-matched nondiabetic subjects. Within diabetic or nondiabetic subjects, the trend for all four parameters to increase in frequency or magnitude in the order neck $<$ thigh $<$ calf was highly significant; the only statistically significant difference between calf and foot muscles for any of the four parameters was capillary basement membrane width for nondiabetic subjects, which was significantly thinner in foot than in calf muscle $(t=2.45$; $p<0.05$ ). Pericyte coverage of capillaries did not differ between diabetic and nondiabetic subjects for each muscle examined; however, capillary basement membrane width, the frequency of pericyte debris and acellular capillaries were in-
\end{abstract}

creased significantly in the lower extremity muscles of diabetic compared to nondiabetic subjects, and the magnitude of the difference between these two groups increased in the order thigh $<$ calf $<$ foot. The observations that pericyte degeneration and acellular capillaries are present in skeletal muscle as well as in retinal microvessels suggest that common pathophysiological mechanisms may contribute to vascular disease in these two very different tissues. The additional finding that relative differences between diabetic and nondiabetic subjects, in the frequency and magnitude of these changes, increase in the order neck $<$ calf $<$ foot is consistent with the marked increase in peripheral vascular disease and gangrene in the lower extremities of diabetic patients.

Key words: Pericyte, diabetes, acellular capillaries, skeletal muscle, diabetic retinopathy, capillary basement membrane width.
Capillary degenerative changes associated with diabetes have been well documented in the retina and include microaneurysms, basement membrane thickening, loss of pericytes, capillary closure (acellular capillary basement membranes), capillary shunting, neovascularization, and related extravascular changes such as haemorrhages and exudates [1-4]. Except for capillary basement membrane thickening, there is a paucity of information regarding the frequency of these degenerative phenomena in diabetic tissues other than the retina. We have noted previously the presence of acellular capillaries and cellular debris consistent with pericyte necrosis in quadriceps femoris and gastrocnemius muscles of human diabetic subjects [5]. However, there is virtually no quantitative information regarding the frequency of these phenomena in the diabetic foot, which is particularly susceptible to the diabetic complications of vascular disease and neuropathy, and in which the frequency of gangrene is many times that of the nondiabetic population [6]. The present study was undertaken to quantify the relative frequency of pericyte degeneration and acellular capillaries in selected skeletal muscles of human diabetic and nondiabetic subjects, including those in the foot.

\section{Materials and methods}

\section{Selection of subjects}

Neck (sternocleidomastoid), thigh (quadriceps femoris), calf (gastrocnemius), and foot (abductor hallicus) muscles were obtained at autopsy from 5 male and 4 female diabetic subjects and 9 age- and sex-matched nondiabetic controls with postmortem intervals of less than $12 \mathrm{~h}$. Diabetic subjects (see Table 1 ) were selected on the basis of a history of diabetes, elevated blood glucose values, and light microscopic documentation of diffuse or nodular diabetic glomerulosclerosis. Since it is well known that some diabetic patients do not develop any manifestations of diabetic vascular disease regardless of the duration of diabetes, this criterion was employed to ensure that the individuals selected for study had documented evidence of their susceptibility to develop diabetic vascular disease. Control subjects were selected on the basis of a negative history of diabetes or normal blood glucose values. 
Table 1. Diabetic and control subjects

\begin{tabular}{|c|c|c|c|c|c|c|c|c|c|c|c|c|}
\hline \multirow{2}{*}{$\begin{array}{l}\text { Patient } \\
\text { number }\end{array}$} & \multirow{2}{*}{$\begin{array}{l}\text { Age } \\
\text { (year) } \\
\& \text { sex }\end{array}$} & \multirow{2}{*}{$\begin{array}{l}\text { Duration } \\
\text { diabetes } \\
\text { (years) }\end{array}$} & \multirow{2}{*}{$\begin{array}{l}\mathrm{BG}^{2} \\
(\mathrm{mg} / \mathrm{dl})\end{array}$} & \multicolumn{4}{|c|}{ Vessels evaluated ${ }^{3}$} & \multirow{2}{*}{$\begin{array}{l}\text { Type of } \\
\text { diabetes }\end{array}$} & \multirow[t]{2}{*}{ Treatment } & \multirow[t]{2}{*}{ AVD $^{4}$} & \multirow{2}{*}{$\begin{array}{l}\text { Hyper- } \\
\text { tension }^{5}\end{array}$} & \multirow{2}{*}{$\begin{array}{l}\text { Diabetic } \\
\text { complications }\end{array}$} \\
\hline & & & & $\mathbf{N}$ & $Q$ & $\mathrm{G}$ & $\mathbf{F}$ & & & & & \\
\hline \multicolumn{13}{|c|}{ Diabetic subjects } \\
\hline 1 & $35 \mathrm{f}$ & 25 & $416 \mathrm{r}$ & 11 & 13 & 16 & - & I & Insulin & 2 & + & $1,2(\mathrm{~d}), 4$ \\
\hline 2 & $64 f$ & 25 & $384 \mathrm{r}$ & 20 & 20 & 19 & 17 & II & Insulin & 2 & + & $1,2(\mathrm{n}, \mathrm{d})$ \\
\hline 3 & $81 \mathrm{f}$ & 3 & $247 \mathrm{r}$ & 16 & 16 & 17 & 14 & - & Insulin & 3 & + & $2(n, d)$ \\
\hline $4^{1}$ & $85 \mathrm{f}$ & 20 & $849 r$ & 23 & 22 & 22 & 20 & II & Insulin & 2 & - & 2 (d) \\
\hline $5^{1}$ & $35 \mathrm{~m}$ & 11 & $361 \mathbf{r}$ & 12 & 9 & 18 & 17 & I & Insulin & 2 & + & $2(\mathrm{n}, \mathrm{d})$ \\
\hline 6 & $62 \mathrm{~m}$ & 6 & $271 \mathbf{r}$ & 20 & 21 & 13 & 13 & II & Diet & 2 & + & $2(\mathrm{n}, \mathrm{d})$ \\
\hline 7 & $67 \mathrm{~m}$ & - & $218 r$ & 23 & 18 & 18 & 13 & II & Insulin & 3 & + & $2(\mathrm{n}, \mathrm{d})$ \\
\hline 8 & $67 \mathrm{~m}$ & 4 & $526 \mathrm{r}$ & 20 & 17 & 18 & 22 & II & Insulin & 3 & - & 2 (d) 4 \\
\hline 9 & $89 \mathrm{~m}$ & - & $302 r$ & 9 & 17 & 16 & 13 & II & Oral agent & 2 & + & $2(\mathrm{n}, \mathrm{d}) 3,4$ \\
\hline \multicolumn{13}{|c|}{ Control subjects } \\
\hline 1 & $36 \mathrm{f}$ & & $162 \mathrm{r}$ & 13 & 11 & 17 & 16 & & & - & + & \\
\hline 2 & $64 \mathrm{f}$ & & $223 r$ & 18 & 18 & 18 & 19 & & & - & + & \\
\hline 3 & $80 f$ & & $223 r$ & 13 & 14 & 14 & 13 & & & 3 & + & \\
\hline 4 & $82 \mathrm{f}$ & & $\ldots$ & 19 & 21 & 22 & 18 & & & - & - & \\
\hline 5 & $38 \mathrm{~m}$ & & $99 \mathrm{r}$ & 10 & 9 & 8 & 13 & & & - & - & \\
\hline 6 & $60 \mathrm{~m}$ & & $108 r$ & 13 & 31 & 21 & 17 & & & 3 & + & \\
\hline 7 & $64 \mathrm{~m}$ & & $168 r$ & 12 & 14 & 16 & 11 & & & 3 & + & \\
\hline 8 & $68 \mathrm{~m}$ & & $117 r$ & 25 & 21 & 17 & 17 & & & 2 & + & \\
\hline 9 & $86 \mathrm{~m}$ & & $105 r$ & 17 & 17 & 16 & - & & & 3 & - & \\
\hline
\end{tabular}

${ }^{1}$ Family history of diabetes; ${ }^{2} \mathrm{BG}=$ blood glucose; $\mathrm{r}$ = random value; - no value available; ${ }^{3}$ number of capillaries evaluated for each muscle; $\mathrm{N}=$ neck (sternocleidomastoid); $\mathrm{Q}=$ quadriceps femoris; $\mathrm{G}=$ gastrocnemius; $\mathrm{F}=$ foot (abductor hallicus); ${ }^{4}$ atherosclerotic vascular disease (aorta, coronary, and cerebral arteries) diagnosed at autopsy. $-=$ no evidence, $1=$ slight, $2=$ moderate, $3=$ severe; ${ }^{5}$ based on clinical notation of hypertension and treatment with antihypertensive agents; ${ }^{6} 1=$ retinopathy; 2 =diabetic glomerulosclerosis, nodular (n) and/or diffuse (d); $3=$ hyalinization of islets of Langerhans; $4=$ neuropathy. Retinopathy and neuropathy were diagnosed clinically; nephropathy and islet degenerative changes were documented at autopsy by light microscopy

Table 2. Cause of death in diabetic and control subjects

\begin{tabular}{cll}
\hline $\begin{array}{l}\text { Patient } \\
\text { number }\end{array}$ & Cause of death & Additional comments \\
\hline Diabetic subjects & \\
1 & Myocardial infarction & \\
2 & Spleen puncture & Infarcts in spleen, descending colon, cerebellum \\
3 & Cerebral infarction & Left ventricle hypertrophy, history of renal failure \\
4 & Small bowel infarction & Cardiomegaly, arteriolonephrosclerosis \\
5 & Bacterial endocarditis & Arteriolonephrosclerosis \\
6 & Bronchial pneumonia & Cardiomegaly, acute tubular necrosis \\
7 & Myocardial infarction & Cardiomegaly \\
8 & Pulmonary emboli, bilateral & History of angina and CVA \\
9 & pneumonia & Myocardial infarction \\
& Pneumonia, acute renal tubular necrosis & Left ventricular hypertrophy, arteriolonephrosclerosis \\
Control subjects & & \\
1 & Subarachnoid haemorrhage & Cardiomegaly, right heart failure \\
2 & Gastric ulcer & Fibrosis of both lungs, pulmonary thromboemboli \\
3 & Cerebral infarction & Right internal carotid artery occlusion \\
4 & Brain tumour (lymphoma) & Right hemiparesis \\
5 & Myocarditis & Cardiomegaly \\
6 & Myocardial infarction & Renal infarcts, arteriolonephrosclerosis \\
7 & Haemopericardium & Congestive heart failure \\
8 & Pulmonary thromboembolus & Essential hypertension \\
9 & Myocardial infarction & Arteriosclerosis, renal failure \\
\hline
\end{tabular}

\section{Tissue processing}

Tissue samples were fixed for $12-48 \mathrm{~h}$ with $2.5 \%$ glutaraldehyde containing picric acid in $0.1 \mathrm{~mol} / 1$ cacodylate buffer $(\mathrm{pH}=7.4,500 \mathrm{mos}-$ $\mathrm{mol}$ ), rinsed in buffer, then postfixed with $1 \%$ osmium tetroxide in
$0.1 \mathrm{~mol} / 1$ cacodylate buffer for $2 \mathrm{~h}$. After dehydration through $100 \%$ acetone, tissue was embedded in Durcupan and thin sections were cut on a Sorvall Porter-Blum MT2-B ultramicrotome, mounted on 300 mesh copper grids, stained with uranyl acetate followed by lead citrate, and examined in a JEOL $100 \mathrm{C}$ electron microscope at $60 \mathrm{kv}$. 

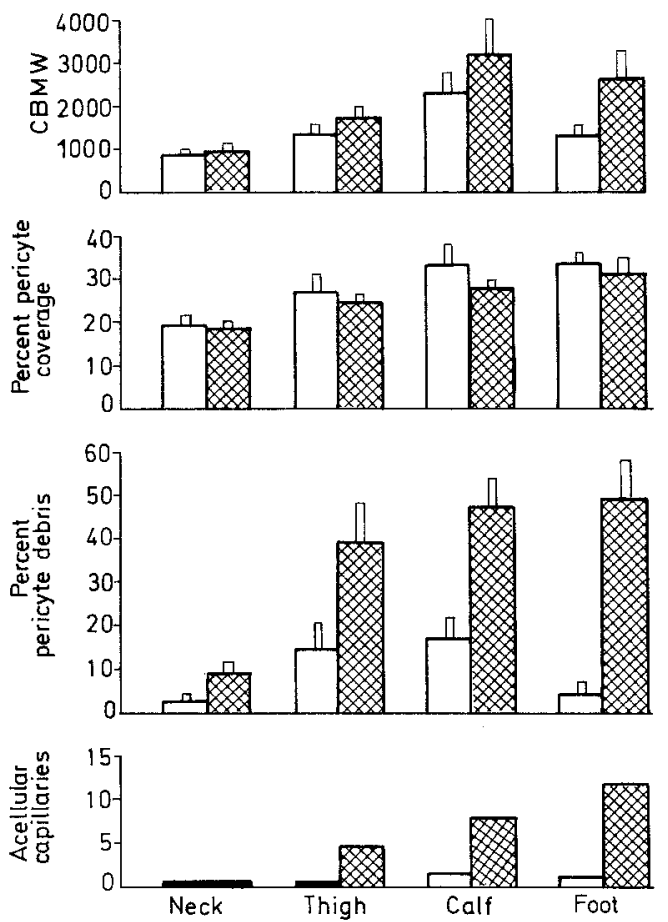

Fig. 1. Capillary basement membrane width (CBMW), percentage of capillary circumference covered by pericyte processes, percentage of capillaries containing cellular debris within basement membranes, and the extent of acellular capillaries (capillary basement membrane remnants devoid of intact endothelial cells and pericytes) expressed as a percent of total number of vessels used for morphometry from each skeletal muscle, for control (open bars) and diabetic (hatched bars) subjects in neck, thigh, calf, and foot. Values represent mean \pm SEM. Vessels formed by more than three endothelial cells and with maximum length to width ratios greater than two were eliminated from morphometry. Females and males were grouped, since no significant sex differences were evident.

Calibration grids were photographed daily for determination of the absolute magnification of each photograph.

\section{Capillary morphometry}

In order to increase the likelihood that true capillaries were examined, only cross-sectioned vessels (maximum length to width ratio less than two) formed by three or fewer endothelial cells and not surrounded by vascular smooth muscle were utilized for morphometry. Vessels with length to width ratios greater than two were considered to be tangentially or longitudinally sectioned, and were excluded from analysis. All vessels meeting the above criteria were photographed ( $\sim 6-12$ capillaries) from each of three different tissue blocks from each skeletal muscle, then coded prior to morphometry. Quantitative estimates of capillary basement membrane width (CBMW) were obtained by the two-point minimum method of Williamson et al. [7]. A Hewlett-Packard $1000 \mathrm{~A}$-series computer and digitizer were used to obtain estimates of: (1) capillary circumference, which was calculated by measuring the total length of the outer endothelial cell membrane, and (2) the percentage of the capillary circumference covered by pericyte processes. The latter was calculated by measuring that portion of the capillary circumference in direct apposition with pericyte processes, then expressing this value as a percent of total capillary circumference.

The presence of cellular debris within capillary basement membranes was tabulated as an index of pericyte necrosis, since pericytes are the only cells in the capillary wall completely ensheathed by basement membrane. As described previously [5], aggregates of cytoplas- mic constituents devoid of intact organelles and not bounded by plasma membrane were considered to represent pericyte debris. All coded vessels that were used for morphometry were evaluated for the presence of debris independently by two observers and discrepancies were arbitrated by a third person. The extent of acellular capillaries within each skeletal muscle was assessed by expressing the total number of capillary basement membranes (in cross section) devoid of intact endothelial cells and pericytes from every tissue block sampled as a percentage of the total number of capillaries that were used for morphometry. No morphometry was performed on these vessels.

\section{Statistical analysis}

A mean and SEM were determined for each parameter assessed for each skeletal muscle, where $n$ represented numbers of patients evaluated. Females and males were grouped, since no sex differences were evident. A repeated-measures analysis of variance (ANOVA) test was performed according to diagnosis (diabetic versus nondiabetic subjects) and muscle location. An analysis procedure (Statistical Analysis System; SAS Institute, Cary, NC, USA) also was used to assess the trend for the difference in capillary basement membrane width, pericyte debris and capillary necrosis between diabetic and nondiabetic subjects to increase in the direction of neck to foot.

\section{Results}

\section{Clinical data}

Diabetic subjects (Table 1) ranged in age from 35 to 89 years; based on clinical records, these subjects had a mean duration of diabetes of $13.4 \pm 9.7$ (SD) years ( 3 to 25 years). Six of nine diabetic subjects had Type 2 (noninsulin-dependent) diabetes; seven of nine subjects were maintained on insulin therapy with one subject treated with diet and one with an oral agent. Moderate to severe atherosclerotic vascular disease (aorta, coronary and cerebral arteries) was confirmed at autopsy in all diabetic subjects and hypertension was noted clinically in seven subjects. Systolic and diastolic blood pressures were $167 \pm 38$ (SD) $\mathrm{mmHg}$ and $97 \pm$ $16 \mathrm{mmHg}$, respectively, in the hypertensive diabetic subjects. Diffuse or nodular diabetic glomerulosclerosis was confirmed by light microscopy in all diabetic subjects. Retinopathy was documented clinically in two diabetic subjects and neuropathy in three. Myocardial, small bowel and cerebral infarctions were the cause of death in 4 of 9 diabetic subjects; three died from pneumonia and one from bacterial endocarditis.

Slight arteriolonephrosclerosis was confirmed with light microscopy in two nondiabetic subjects, while one subject (Table 1, No.8) exhibited marked arterial and arteriolar nephrosclerosis; the renal vasculature of the remainder of the nondiabetic subjects was normal. Moderate to severe atherosclerotic vascular disease (aorta, coronary and cerebral arteries) was confirmed at autopsy in 5 of 9 nondiabetic subjects, while four subjects (predominantly females) had no evidence of atherosclerosis. Hypertension was noted clinically in 6 of 9 nondiabetic subjects and systolic and diastolic blood pressures were $167 \pm 34 \mathrm{mmHg}$ and $97 \pm 17 \mathrm{mmHg}$ respectively. Cardiovascular disease was the major cause of death in the same number of subjects (Table 2). 


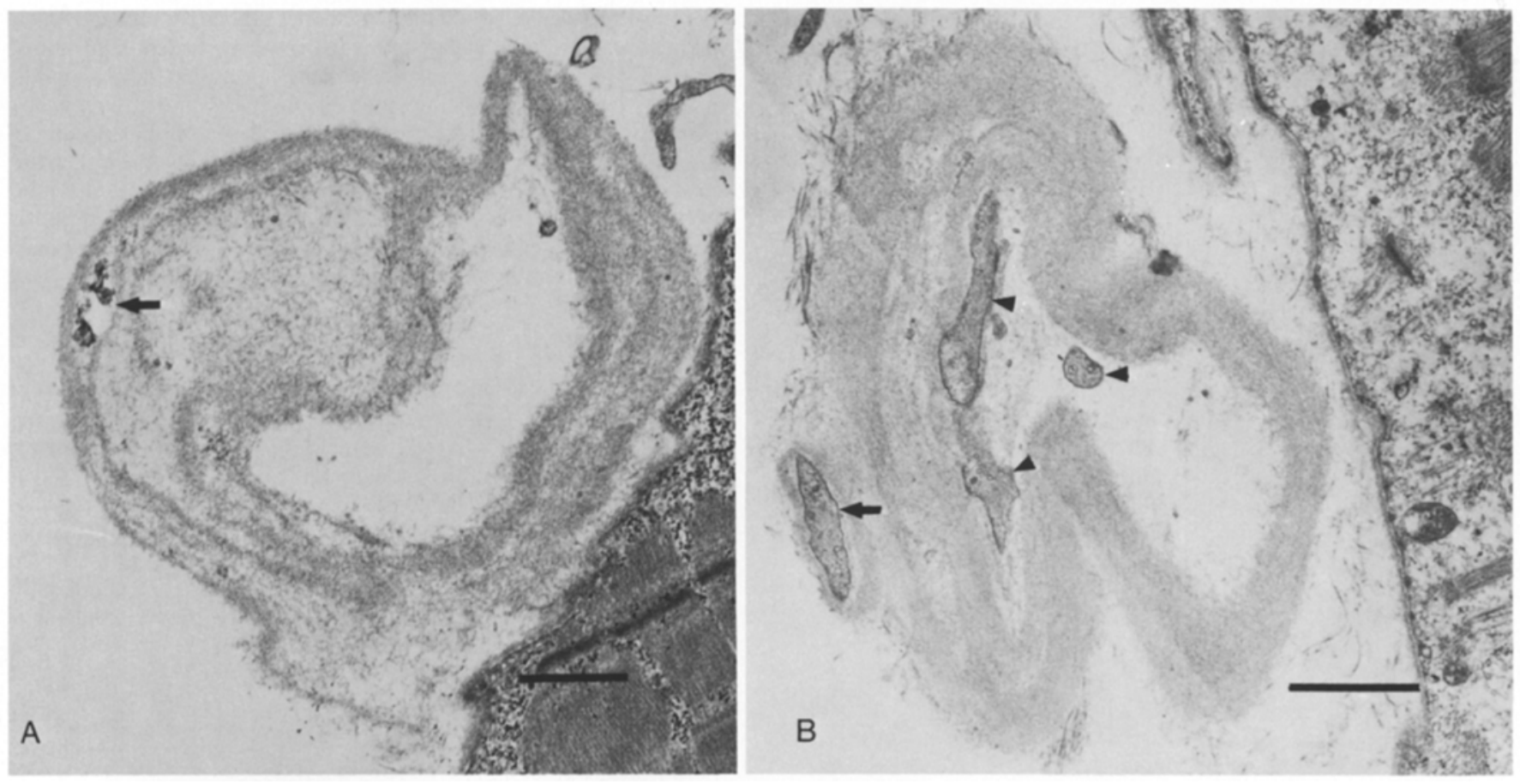

Fig. 2. Cross-sectioned capillaries obtained at autopsy from muscle of diabetic subject. A Endothelium has been completely denuded from the lumenal side of the basement membrane and only a small accumulation of membranous vesicular cell debris remains within the basement membrane (arrow). $\times 13,300$; bar represents $1 \mu \mathrm{m}$. B An intact pericyte process (arrow) is present within the capillary basement membrane, but only a small portion of the lumenal circumference is covered by an intact cell (arrowheads), $\times 15,900$; bar represents $1 \mu \mathrm{m}$

The trend for mean capillary basement membrane width (CBMW) to increase in the direction of neck to foot (Fig. 1) was significant for both diabetic subjects (ANOVA; $\mathrm{F}=54.2, p<0.0001$ ) and nondiabetic subjects (ANOVA; $\mathrm{F}=16.2, p<0.0002$ ) even though mean values in both groups were higher in the calf than in the foot. Although CBMW was consistently thicker in diabetic than in nondiabetic subjects in lower extremity skeletal muscles, differences were statistically significant only in thigh and foot (Wilcoxon rank-sum test; $p<0.025$ for both thigh and foot muscles). The trend for the magnitude of the difference between diabetic and nondiabetic subjects to increase in the direction of neck to foot also was significant $(\mathrm{F}=5.41, p<0.03)$.

Capillary circumference ranged from 17.6 to $19.9 \mu \mathrm{m}$ (data not shown) and did not differ between females and males or between diabetic and nondiabetic subjects in the four skeletal muscles examined. The percentage of capillary circumference covered by pericytes (Fig. 1) increased in the order neck $<$ thigh $<$ calf $<$ foot for diabetic (ANOVA; $\mathrm{F}=10.1, p<0.003$ ) and nondiabetic subjects (ANOVA; $\mathrm{F}=28.2, p<0.0001$ ), and did not differ between the two groups or between females and males within each group. In nondiabetic subjects, cell debris within capillary basement membranes was present in less than $5 \%$ of capillaries sampled from neck and foot (Fig. 1) and was present in $15-17 \%$ of capillaries sampled from thigh and calf. Cell debris was much more prevalent in capillary basement membranes of diabetic subjects, increasing from $9 \%$ in neck to $47-49 \%$ in calf and foot (ANOVA; $F=40.3$, $p<0.0001$ ). In addition, multiple foci of debris often were present in individual vessels of diabetic subjects. The trend for the difference in the frequency of cell debris between diabetic and nondiabetic subjects to increase in the direction of neck to foot was highly significant $(\mathrm{F}=18.4, p<0.0003)$.

In nondiabetic subjects, acellular capillaries were not observed in neck and thigh muscles and represented $\sim 1 \%$ of capillaries sampled from calf and foot. The frequency of acellular capillaries was increased in lower extremity muscles of diabetic relative to nondiabetic subjects, and the trend for acellular capillaries to increase in the direction of neck to foot in diabetic subjects was significant (ANOVA; $\mathrm{F}=6.60, p<0.01$ ). An example of an acellular capillary is shown in Figure $2 \mathrm{~A}$; note the absence of endothelium on the lumenal side of the basement membrane and only a small accumulation of debris, presumably of pericyte origin, within the basement membrane (arrow). In Figure 2B, an intact pericyte process (arrow) and 2-3 unidentifiable cell processes (endothelium or pericyte) covering a small portion of the lumenal side of the basement membrane (arrowheads) are the only remaining cellular elements of this capillary.

\section{Discussion}

Our use of multiple tissue biopsies from each human subject limited this study to autopsy tissue. This approach appeared valid, however, since tissue obtained 
postmortem and fixed by immersion has been used extensively to measure capillary basement membrane width. In addition, we assessed only capillary degenerative changes (necrotic cellular debris and acellular capillaries) which would be manifested after days rather than hours of cellular autolysis, and we avoided quantification of lumenal and endothelial cell areas, which would be influenced by intracellular swelling. While the maximum postmortem interval was limited to $12 \mathrm{~h}$, the mean postmortem duration was much less for both diabetic $(8.2 \pm 4.2(\mathrm{SD}) \mathrm{h})$ and nondiabetic $(7.8 \pm 3.4 \mathrm{~h})$ subjects. No differences were evident in the rate of autolysis of tissues from diabetic and nondiabetic subjects with comparable postmortem intervals as judged from the quality of preservation of ultrastructural detail.

The finding in this study that cellular debris within capillary basement membranes, undoubtedly derived from pericytes, is increased significantly in lower extremity skeletal muscles of diabetic compared to nondiabetic subjects is consistent with several previous reports in skeletal muscle $[5,8]$ and skin $[9,10]$. The observation that pericyte circumferential coverage of capillaries does not differ between diabetic and nondiabetic subjects within each skeletal muscle suggests that pericyte turnover is increased, but that regeneration keeps pace with accelerated degeneration. This observation further suggests that the diabetic milieu injures capillary pericytes in skeletal muscle, but that the homeostatic mechanisms governing the relative proportions of these cells are not disturbed, or that endothelial cell and pericyte regenerative capacity has not been compromised or exhausted. This is in contrast to the retina, where pericytes are selectively lost in diabetes and are absent at sites of aneurysm formation $[1,2]$.

While capillaries devoid of intact cells and consisting merely of basement membrane tubes were first demonstrated in the retina over 30 years ago [11] and subsequently confirmed in vivo by fluorescein angiography [12], the cause and pathophysiological significance of this phenomenon remain unclear today. Several hypotheses have been advanced to explain the pathogenesis of acellular capillaries in the retina, including arteriolar hyalinization and occlusion [11] and capillary compression caused by increased retinal edema resulting from tissue metabolic disturbances [13]. It also has been suggested that acellular capillaries are the end result of capillary haemodynamic changes produced by pericyte loss, microaneurysm formation and capillary arteriovenous shunts [2]. Arteriolar narrowing and occlusion may be an important factor in the pathogenesis of acellular capillaries in the retina since hyaline arteriolosclerosis and arteriolar obliteration have been reported in retinas of diabetic subjects [14], and focal retinal ischemia has been considered to be a prominent feature of diabetic retinopathy $[15,16]$. These vessel remnants are probably nonfunctional in the retina, since they do not contain erythrocytes [2] and are not perfused in fluorescein angiographic studies [12, 17-19].
The demonstration in this study of acellular capillaries in lower extremity skeletal muscles is consistent with at least two previous reports of this phenomenon in skeletal muscle $[8,20]$. In the study by Vracko [8], eight different muscle sites were sampled (from chest to foot), but not all muscles were obtained from every patient and no quantitative data on the extent of acellular capillaries within each muscle were provided. Although the presence of intact cell processes within capillary basement membrane remnants (Fig. 2B) suggests that endothelial cells and pericytes may revascularize acellular capillaries, as previously suggested by Vracko [8], it should be noted that virtually all acellular capillaries observed in this study were devoid of intact cells.

The presence of pericyte degeneration and acellular capillaries in both retina and skeletal muscle suggests that common pathophysiological mechanisms may be responsible for the vascular complications of diabetes in these two very different tissues. Since generalized arteriolosclerosis and occlusion of medium-sized and smaller muscular arteries are much more pronounced in diabetic subjects than in the general population [21], hyalinization, narrowing, and obliteration of precapillary arterioles in skeletal muscle, as in the retina, could result in chronic ischemia leading to necrosis and atrophy of the more distal microvasculature. The chronic ischemia may stimulate angiogenesis $[15,22]$ which is not beneficial to the retina (the capillary proliferation occurs on or near the surface of the retina, predisposing the eye to preretinal haemorrhage and retinal detachment), but which may salvage skeletal muscle tissue.

The trend for capillary basement membrane thickening, pericyte degeneration, and acellular capillaries to increase in the direction of neck to foot in diabetic (and nondiabetic) subjects, also suggests that these pathological phenomena may be related to increases in venous blood pressure. Although it is unclear how venous hydrostatic pressure would influence pericyte and endothelial cell degeneration and turnover, it is of interest that venous dilatation is observed clinically early in the course of diabetic retinopathy [3].

While metabolic perturbations or focal alterations in the structural or functional integrity of endothelial cells may lead to microthrombi and ultimately to capillary necrosis and acellular capillaries, the presence of confirmatory intralumenal clots and haemorrhage is virtually impossible to assess with ultrastructural techniques. On the other hand, several recent studies have raised the possibility that increased metabolism of glucose by the enzyme aldose reductase within pericytes may lead to increased pericyte degeneration and necrosis. Aldose reductase has been demonstrated immunohistochemically [23] and localized by radioimmunoassay [24] within retinal pericytes, and cultured retinal pericytes incubated in a high glucose medium accumulate sorbitol [24]. In the latter study, extensive cellular debris was evident after pericytes were incubated in the high glucose medium for 30 days. Since we have shown 
previously that skeletal muscle capillary pericytes in rats contract when stimulated with vasoconstricting agents [25], metabolic perturbations which affect the contractile function or the structure of these cells could result in haemodynamic changes which, in turn, might contribute to endothelial injury, ultimately leading to acellular capillaries. It should be noted, however, that the physiological function of retinal pericytes is poorly understood and there are no published reports that skeletal muscle capillary pericytes contain aldose reductase.

We have no explanation for the finding that capillary basement membranes are thinner in the foot than in calf muscle. Although the decrease was significant only in nondiabetic subjects (paired t-test; $t=2.45, p<0.05$ ), the fact that differences were evident in both groups gives credence to the finding. A possible explanation which would be consistent with the postulated relationship between venous hydrostatic pressure and capillary basement membrane width in the other muscles examined is that hydrostatic pressure in the vasculature of the foot may be counterbalanced by a much higher external pressure exerted on these vessels in subjects standing or walking.

In conclusion, these findings indicate that pericyte degeneration and acellular capillaries are generalized phenomena in diabetes. The observation that these capillary degenerative phenomena are more prevalent in the foot than in the neck and trunk is consistent with the accelerated vascular disease and gangrene in the lower extremity of diabetic patients.

Acknowledgements. We thank S. Brickey for her excellent secretarial skills, J. Fulford for his photographic assistance, and J. Howe for his help with the capillary morphometry. This study was supported in part by grants from the National Institutes of Health (HL30678, HL13694, AM20579) and by the Kilo Diabetes and Vascular Research Foundation.

\section{References}

1. Cogan DG, Toussaint D, Kuwabara T (1961) Retinal vascular patterns. IV. Diabetic retinopathy. Arch Ophthalmol 66: 366 378

2. Cogan DG, Kuwabara T (1963) Capillary shunts in the pathogenesis of diabetic retinopathy. Diabetes: $12: 293-300$

3. Garner A (1970) Pathology of diabetic retinopathy. Br Med Bull 26: $137-142$

4. Yanoff M (1969) Ocular pathology of diabetes mellitus. Am J Ophthalmol 67:21-38

5. Tilton RG, Hoffmann PL, Kilo C, Williamson JR (1981) Pericyte degeneration and basement membrane thickening in skeletal muscle capillaries of human diabetics. Diabetes 30:326-334
6. Bell ET (1957) Atherosclerotic gangrene of the lower extremities in diabetic and nondiabetic persons. Am J Clin Pathol 28: 27-36

7. Williamson JR, Vogler NJ, Kilo C (1969) Estimation of vascular basement membrane thickness: theoretical and practical considerations. Diabetes 18: $567-578$

8. Vracko R (1970) Skeletal muscle capillaries in diabetics. A quantitative analysis. Circulation 41 : 271-283

9. Friederici AHR, Tucker WR, Schwartz TB (1966) Observations on small blood vessels of skin in the normal and in diabetic patients. Diabetes 15: 233-250

10. Yodaiken RE, Seftel HC, Rubenstein AN (1967) Ultrastructure of dermal capillaries of Africans in South Africa. Diabetes 16: 191-197

11. Ashton $\mathbf{N}$ (1953) Central areolar choroidal sclerosis. A histopathological study. Br J Ophthalmol 37: 140-147

12. Kohner EM, Dollery CT, Paterson JW, Oakley NW (1967) Arterial fluorescein studies in diabetic retinopathy. Diabetes 16:1-10

13. Ashton N (1959) Diabetic retinopathy. A new approach. Lancet 2: 625-630

14. Ashton $N$ (1953) Arteriolar involvement in diabetic retinopathy. Br J Ophthalmol 37: 282-292

15. Ashton N (1961) Neovascularization in ocular tissue. Trans Ophthalmol Soc UK 81 : $145-161$

16. Bresnick GH, de Venecia G, Myers FL, Harris JA, Davis MD (1975) Retinal ischemia in diabetic retinopathy. Arch Ophthalmol 93: $1300-1310$

17. Scott DJ, Dollery CT, Hill DW, Hodge JV, Fraser R (1964) Fluorescein studies of diabetic retinopathy. $\mathrm{Br}$ Med J 1: 811-814

18. Oosterhuis JA, Vink R (1968) Fluorescein photography in diabetic retinopathy. In: Henkes $H$ (ed) Perspectives in Ophthalmology. Excerpta Medical Foundation, Rotterdam, pp 115-132

19. Kohner EM, Henkind $\mathbf{P}$ (1970) Correlation of fluorescein angiogram and retinal digest in diabetic retinopathy. Am J Ophthalmol 69: $403-414$

20. Zacks SI, Pegues JJ, Elliott FA (1962) Interstitial muscle capillaries in patients with diabetes mellitus: a light and electron microscopic study. Metabolism 11: 381-393

21. Williamson JR, Kilo C, Crespin SR (1977) Vascular disease. In: Levin ME, O'Neal LW (eds) The Diabetic Foot. C.V.Mosby, St. Louis, pp 67-96

22. Kumar S, West D, Shahabuddin S, Arnold F, Haboubi N, Reid H, Carr T (1983) Angiogenesis factor from human myocardial infarcts. Lancet 2: 364-367

23. Akagi Y, Kador PF, Kuwabara T, Kinoshita JH (1983) Aldose reductase localization in human retinal mural cells. Invest Ophthalmol Vis Sci 24: 1516-1519

24. Buzney SM, Frank RN, Varma SD, Tanishima T, Gabbay KH (1977) Aldose reductase in retinal mural cells. Invest Ophthalmol Vis Sci 16: $392-396$

25. Tilton RG, Kilo C, Williamson JR, Murch DW (1979) Differences in pericyte contractile function in rat cardiac and skeletal muscle. Microvase Res 18: 336-352

Received: 28 May 1985

and in revised form: 18 September 1985

Dr. Ronald G. Tilton

Washington University School of Medicine

Department of Pathology

660 S. Euclid Avenue

St. Louis, MO 63110

USA 